\title{
Derzeitiger Stellenwert der adjuvanten medikamentösen Ovarektomie
}

\author{
R. Jakesz im Namen der Austrian Breast and Colon Cancer Study Group (ADCSG) \\ Universitätsklinik für Chirurgie - Klinische Abteilung für Allgemeinchirurgie AKH Wien
}

Die adjuvante Therapie von prämenopausalen Patientinnen mit Mammakarzinom war bisher eine Domäne der Chemotherapie. Overview-Daten über adjuvante Chemotherapie zeigen eine 35\%ige Verminderung der Rezidive und eine 27\%ige Verringerung der Todesrate durch verschiedene Polychemotherapieschemata. Die lokale Ausschaltung der Ovarialfunktion durch chirurgische oder radiotherapeutische Ovarektomie konnte jedoch auf ähnliche Effekte verweisen und seit dem letzten Overview über die Wirkung von Tamoxifen ist bekannt, daß diese Substanz, gegeben für mindestens 5 Jahre, bei rezeptorpositiven prämenopausalen Patientinnen zu einer $42 \%$ igen Verbesserung der Rezidivrate führt.

Auf dem ASCO-Meeting 1999 in Atlanta wurden insgesamt 3 Studien vorgestellt, die den Effekt von Goserelin in einem LHRH-Analog in verschiedenen Designs untersuchten (Tab.1). Davidson präsentierte eine Untersuchung an mehr als 1.500 prämenopausalen Patientinnen mit rezeptorpositivem Mammakarzinom und axillären Lymphknotenmetastasen. Diese Untersuchung war eine dreiarmige Studie mit a) einer anthrazyklinhaltigen Basischemotherapie (Cyclophosphamid, Adriamycin, 5-Fluorouracil; CAF), b) dieser Therapie zusätzlich mit Tamoxifen, und c) der Tripelkombination CAF, Tamoxifen und Goserelin. Nach 5 Jahren medianer Nachbeobachtungszeit zeigte sich im Gesamtüberleben kein Unterschied dieser drei Gruppen, jedoch eine signifikante Verbesserung des rezidivfreien Überlebens von 70 auf $78 \%$ durch die Kombination CAF, Tamoxifen und Goserelin, verglichen mit der Basischemotherapie allein. Eine alleinige Goserelingabe zusätzlich zur Basischemotherapie zeigte keine Verbesserung des rezidivfreien Überlebens. Diese Studie läßt den Schluß zu, daß diese Chemotherapie in erster Linie durch die dadurch induzierte Ovarektomie ihre Wirkung ausübt, da eine zusätzliche medikamentöse Ovarektomie in Form von Goserelin keinen wesentlichen, weiteren positiven Effekt erzielen kann. Die zusätzliche Gabe eines Antiöstrogens führt jedoch zu einer deutlichen Verbesserung des rezidivfreien Intervalls, was die Bedeutung der antiöstrogenen Therapie bei prämenopausalen Patientinnen mit rezeptorpositivem Mammakarzinom deutlich unterstreicht und in gute Übereinstimmung zu den TamoxifenDaten im Overview paßt.
Unsere eigene Studie an über 1.000 Patienten dient der Fragestellung, welcher Stellenwert einer kombinationsendokrinen Therapie mit Goserelin und Tamoxifen gegenüber der derzeitigen Standardtherapie mit CMF (Cyclophosphamid, Methotrexat, 5-Fluorouracil) zukommt. In diese Untersuchung konnten auch Patientinnen ohne Lymphknotenmetastasen aufgenommen werden. Nach einer medianen Nachbeobachtungszeit von 4 Jahren entwickelten $16 \%$ aller aufgenommenen Patientinnen ein Rezidiv, 6,5\% der Patientinnen starben. Beim Vergleich des rezidivfreien Überlebens zeigte sich eine statistisch signifikante Verbesserung der Überlebensraten von Patientinnen, die mit der Antihormontherapie behandelt wurden, gegenüber der CMF-Chemotherapie. In der Überlebensrate konnte lediglich ein Trend zugunsten der Hormontherapie festgestellt werden. Patientinnen tolerierten ihre Hormontherapie wesentlich besser und konnten zu $84 \%$ mit voller Dosis behandelt werden, verglichen mit nur $78 \%$ aller Patientinnen der CMF-Therapiegruppe. Patientinnen, die unter $\mathrm{CMF}$ amenorrhoisch wurden, zeigten eine statistisch signifikante Verbesserung der rezidivfreien und Gesamtüberlebensrate.

Die dritte Studie ergab sich durch einen Zusammenschluß mehrerer randomisierender Gruppen und ist durch das pragmatische Randomisierungsdesign weniger homogen als die beiden anderen Studien. 2.631 Patientinnen konnten in 4 Therapiearme randomisiert werden, eine zusätzliche Chemotherapie war erlaubt. Eine unbehandelte Kontrollgruppe wurde einer Tamoxifen- bzw. einer Goserelingruppe und einer Kontrollgruppe gegenübergestellt. Die Auswertung im Rahmen des $2 \times 2$-Designs nach Zoladex ${ }^{\circledR}$ versus kein Zoladex ergab eine statistisch signifikante Verbesserung des rezidivfreien Überlebens der Patienten, die Goserelin erhalten hatten. Das Gesamtüberleben war auch hier erwartungsgemäß noch nicht signifikant unterschiedlich. Bemerkenswerterweise zeigte sich in der Studie von Rutqvist und in unseren eigenen Daten eine signifikante Verringerung der Lokalrezidivrate und der Anzahl des kontralateralen Zweitkarzinoms in praktisch demselben Ausmaß.

Insgesamt lassen diese 3 Studien die Interpretation zu, daß die Kombination Goserelin und Tamoxifen bei prämenopausalen

\begin{tabular}{ll}
\hline KARGER & (1) 1999 S. Karger GmbH, Freiburg \\
Fax +49 761 4520714 & Accessible online at: \\
$\begin{array}{l}\text { E-mail kargergmbh@aol.com } \\
\text { www.karger.com }\end{array}$ & http://BioMedNet.com/karger \\
\end{tabular}

Prof. Dr. R. Jakesz

Universitätsklinik für Chirurgie -

Klinische Abteilung für Allgemeinchirurgie AKH Wien

Währinger Gürtel 18-20,

A-1090 Wien (Österreich) 
Tab. 1. Studien mit Goserelin (Zoladex), präsentiert auf dem ASCO-Meeting 1999 in Atlanta

\begin{tabular}{lllll}
\hline Autor & $\begin{array}{l}\text { Patienten } \\
\mathrm{n}\end{array}$ & Patientenselektion & Design & Ergebnis \\
\hline Davidson & 1504 & Stadium II, HR+ & $\begin{array}{l}\text { CAF } \\
\text { CAF+TAM } \\
\text { CAF+TAM+GOS }\end{array}$ & $\begin{array}{l}\text { für RFS: CAF vs. } \\
\text { CAF+TAM+GOS } \\
\mathrm{p}<0,05\end{array}$ \\
& & & CMF & $\begin{array}{l}\text { TAM+GOS vs. CMF } \\
\mathrm{p}=0,05\end{array}$ \\
Jakesz & 1045 & Stadien I+II, HR+ & TAM+GOS & GOS vs. KO \\
& & & GOS & \\
Rutqvist & 2631 & Stadien I+II & TAM & GOS+TAM KO \\
& & & KO & \\
& & & &
\end{tabular}

HR $+=$ Hormonrezeptor; $\mathrm{CAF}=$ Cyclophosphamid, Adriamycin, 5-Fluorouracil;

TAM = Tamoxifen; GOS = Goserelin CMF = Cyclophosphamid, Methotrexat, 5-Fluorouracil; RFS = rezidivfreies Überleben; $\mathrm{KO}=$ Kontrolle
Patientinnen mit rezeptorpostivem Mammakarzinom eine wirkungsvolle Therapie ist und einer adjuvanten Chemotherapie zumindest ebenbürtig, wenn nicht überlegen. Auch die zusätzliche endokrine Kombinationstherapie zur Chemotherapie ist erfolgversprechend. Trotzdem müssen längere Nachbeobachtungszeiten abgewartet werden, um einen Einblick in den Effekt dieser Therapiekombination auf das Gesamtüberleben zu erhalten. 